Владимир Л. Евсеев, Руфия Ш. Садекова

ПРОТИВОДЕЙСТВИЕ КИБЕРБУЛЛИНГУ В СОЦИАЛЬНЫХ СЕТЯХ

\author{
Владимир Л. Евсеев ${ }^{1}$, Руфия Ш. Садекова ${ }^{2}$ \\ ${ }^{1}$ Российский государственный университет нефти и газа \\ (национальный исследовательский университет) им. И.М. Губкина \\ Ленинский пр-кт, 65, корпус 1, Москва, 119991, Россия \\ ${ }^{2}$ Финансовый университет при Правительстве Российской Федераџии \\ (Финансовый университет), \\ Ленинградский пр-кт, 49, Москва, 125993, Россия \\ le-mail: Evll@list.ru, https://orcid.org/0000-0003-3283-3106 \\ ${ }^{2}$ e-mail: sadekova.rufiya@gmail.com,https://orcid.org/0000-0001-6223-7730

\section{ПРОТИВОДЕЙСТВИЕ КИБЕРБУЛЛИНГУ В СОЦИАЛЬНЫХ СЕТЯХ DOI: http://dx.doi.org/10.26583/bit.2021.3.08}

Аннотация. Статья посвящена разработке программы определения тональности текста. В статье обосновывается актуальность защиты социума от кибербуллинга. Анализируются методы противодействия кибербуллингу. Введен показатель негативности информации сайта. Подробно рассмотрена работа блокировщика сайтов. Обосновано использование сентимент-анализа, в основе которого лежит использование нейронных сетей. Для сентимент-анализа информационных потоков разработана программа на высокоуровневом языке программирования Python c внедрением в нее готовых обученных нейронных сетей. Используется словарь по стемам. Информационные потоки разбиты на токены, представленные в виде векторов. Приведены примеры нейронных сетей для определения тональности текста. Проведено сравнение результатов выполнения кодов анализа текста по вероятности получения правильного уровня негативности текста. Обосновывается целесообразность использования блокировщиков сайтов, как методов для защиты от кибербуллинга, и использование датасетов для обучения нейронных сетей.

Ключевые слова: кибербуллинг, блокировщик сайтов, межсетевой экран, негативная информация, анализ тональности текста, сентимент-анализ, нейронные сети, стемы.

Для иитирования: ЕВСЕЕВ, Владимир Л.; САДЕКОВА, Руфия Ш. ПРОТИВОДЕЙСТВИЕ КИБЕРБУЛЛИНГУ В СОЦИАЛЬНЫХ СЕТЯХ. Безопасность информаџионных технологий, [S.l.], m. 28, № 3, c. 92-102, 2021. ISSN 2074-7136. URL: https://bit.mephi.ru/index.php/bit/article/view/1366. DOI: http://dx.doi.org/10.26583/bit.2021.3.08.

\author{
Vladimir L. Evseev ${ }^{1}$, Rufiya Sh. Sadekova ${ }^{2}$ \\ ${ }^{1}$ National University of Oil and Gas «Gubkin University» (Gubkin University), \\ Leninsky Prospekt, 65, building 1, Moscow, 119991, Russia \\ ${ }^{2}$ Financial University under the Government of the Russian Federation (Financial University), \\ Leningradsky Prospekt, 49, Moscow, 125993, Russia \\ ${ }^{1}$ e-mail: Evll@list.ru, https://orcid.org/0000-0003-3283-3106 \\ ${ }^{2}$ e-mail: sadekova.rufiya@gmail.com, https://orcid.org/0000-0001-6223-7730
}

\title{
Countering cyberbullying in social networks
}

DOI: http://dx.doi.org/10.26583/bit.2021.3.08

Abstract. The study is devoted to the development of a program for determining the text tonality. The paper substantiates the relevance of protecting society from cyberbullying. The methods of cyberbullying countering are analyzed. An indicator of the negativity of the site's information was introduced. The work of the site blocker is considered in detail. The use of sentiment analysis, which is based on the use of neural networks, is justified. For the sentiment analysis of information flows, a program has been developed in the high-level programming language Python based of ready-made trained neural networks. The stem dictionary is used. Information flows are divided into tokens represented as vectors. In detail, the examples show the use of various neural networks to determine the tonality of the text. The results of the two text analysis codes are compared using the probability of obtaining the correct level of negativity 
of the text. The expediency of using site blockers as methods to protect against cyberbullying as well as the use of datasets for training neural networks are justified.

Keywords: cyberbullying, site blocker, firewall, negative information, sentiment analysis, neural networks.

For citation: EVSEEV, Vladimir L.; SADEKOVA, Rufiya Sh. Countering cyberbullying in social networks. IT Security (Russia), [S.l.], $\quad$ v. 28, n. 3, p. 92-102, $2021 . \quad$ ISSN 2074-7136. URL: https://bit.mephi.ru/index.php/bit/article/view/1366. DOI: http://dx.doi.org/10.26583/bit.2021.3.08.

\section{Введение}

Пандемия коронавируса повлияла не только на экономику, но и на коммуникации в обществе. Межличностное общение вне социальных сетей асимптотически приблизилось к нулю. Общение людей перешло из реального мира в виртуальный мир посредством только социальных сетей. Из-за травли в интернете у многих наблюдается стресс, импульсивность, что наносит вред обществу. И, как результат, во всем мире значительно возросло обращение людей в службы психологической поддержки [1]. Гибридное воздействие злоумышленников на личности возросло на несколько порядков [2]. Одним из его проявлений является кибербуллинг - это агрессия, травля, направленные жертве с помощью средств электронной коммуникации [3]. На государственном уровне встала проблема - как защитить социум от данного негативного воздействия.

Чтобы пользователи социальных сетей не подвергались воздействию кибербуллинга, они должны уметь его распознавать, знать меры защиты от такого воздействия, а государство - должно направлять свои усилия на предотвращение кибербуллинга. С этой целью требуется разработать специальную программу выполняющую анализ тональности текста, позволяющий определять показатели и уровни негативности и являющийся основным элементом блокировщика сайтов.

За 2020 г. кибербуллинг стал одной из самых разрушительных форм онлайн-атак на субъекты. Агрессивные злоумышленники, используя IT-технологии, эмоционально давят на свою жертву, чтобы причинить ей сильнейшую психологическую травму и добиться своих целей [4]. Последствия реализации этих угроз зачастую трагичны и наносят значительный вред обществу.

Как же защищаться от кибербуллера? С нашей точки зрения целесообразно использовать следующие меры:

- организационные меры (общие рекомендации можно взять из программы Калифорнийского университета по борьбе с травлей) [5];

- правовые меры (статьи Уголовного кодекса РФ и Кодекса РФ об административных правонарушениях [6], Федеральные Законы РФ);

- технические меры [7] (компьютерные программы, противодействующие кибербуллингу).

Рассмотрим технические меры защиты от кибербуллинга.

\section{1. Защита интернета от кибербуллинга}

Для защиты пользователей социальных сетей от влияния кибербуллинга целесообразно использовать блокировщик сайтов, в основе которого лежит специальная программа выполняющую анализ тональности текста. Данная программа будет выдавать управляющее воздействие в межсетевой экран для блокировки URL-aдpeca нежелательного сайта. Рассмотрим работу подобной программы, написанной на объектноориентированном высокоуровневом языке программирования Python, ориентированного на обеспечение переносимости написанных на нём программ.

Блокировщик сайтов целесообразно использовать, например, в государственных образовательных учреждения, т.к. он позволяет полностью оградить обучающихся от 
негативной информации и предостеречь обучающихся от кибербуллинга во время нахождения их в учебном заведении (они не видят даже URL-адреса нежелательных сайтов).

Кроме специальной программы в блокировщике требуется использовать систему предотвращения вторжений (Intrusion Prevention System, IPS). IPS позволяет формировать и отсылать новые конфигурации в межсетевой экран, на основе которых межсетевой экран будет фильтровать трафик от нежелательного контента, т.е. кибербуллинга.

Работа блокировщика сайтов основывается на определении доли негативной информации сайта. Чтобы определить, какая доля текста является негативной, введем показатель негативности, который будет лежать в диапазоне от 0 до 1 с дискретностью 0,1. Для систематизации результатов работы блокировщика, определим пороги негативности данного показателя, относящиеся к определенным уровням:

- 0 - 0,3 - низкий уровень негативности;

- 0,31 - 0,6 - средний уровень негативности;

- 0,61 - 0,8 - высокий уровень негативности;

- $0,81-1$ - критический уровень негативности.

Блокировщик сайтов работает следующим образом:

На вход блокировщика подается URL-адрес некоторого сайта, контент (текст) которого требуется оценить на наличие негативной информации.

Копируется текст данного сайта.

Происходит анализ тональности текста.

Определяется уровень негативности текста и происходит отнесение его к тому или иному уровню негативности.

Если выявлен средний, высокий или критический уровень негативности, то IPS выдает сигнал в межсетевой экран на блокировку URL-адреса сайта, а затем его адрес заносится в базу запрещенных сайтов программы.

Если же был получен критический уровень негативности текста, то целесообразно заблокировать сайт и на государственном уровне. Для этого необходимо отправить заявку в Роскомнадзор на сайте Единого реестра сайтов, содержащих запрещенную информацию, заполнив форму сообщения вручную.

Подобный порядок действий программы можно сравнить с межсетевым экраном Китайской Народной Республики, который блокирует на государственном уровне любую информацию негативного подтекста [8].

В основе работы разработанной программы блокировщика сайтов лежит анализ тональности текста (сентимент-анализ), позволяющий определять показатели и уровни негативности. Как происходит анализ эмоциональной окраски текста? Остановимся более подробно на работе сентимент-анализа.

В основе сентимент-анализа лежит подход, основанный на машинном обучении. Традиционно в данной сфере выделяют различные задачи обучения, но в анализе тональности обычно используется только обучение с учителем [9]. А этого недостаточно.

За последние годы количество методов машинного обучения значительно возросло, все чаще применяются различные нейронные сети [10].

Для сентимент-анализа информационных потоков была разработана программа на высокоуровневом языке программирования Python с внедрением в нее готового модуля, например, Dostoevsky, в котором используется уже обученная нейронная сеть [11]. Для ее обучения была использована база данных 2018 г. RuSentiment, в которую входят комментарии из социальной сети ВКонтакте [12]. 
Далее, надо определить - какая из баз данных является более эффективной и влияет ли база данных на качество правильного выполнения анализа тональности текста?

Как же в блокировщике сайтов происходит оценка тональности с помощью нейронной сети? Для примера возьмем два предложения, которые могут встречаться в социальных сетях:

1. Я купила новую книгу, но она мне абсолютно не понравилась, ужасный сюжет.

2. Мальчик купил бабушке цветы, это так мило!

По данным предложениям формируем словарь, не по словам, а по стемам (stem основа слова без суффиксов и окончаний), что позволит избегать повторений слов в разных падежах, числах, словоформах (например, стем слова «легендарный» - «легенд»). Также важно учитывать из предложения - какой тональности каждый стем. Это будет учитываться в дальнейшем в нейронной сети. Чтобы рассмотреть, как определяется тональность текста с помощью нейронной сети, для примера возьмем третье предложение - Милый мальчик купил мне книгу. Представим в виде нулевого вектора все стемы из словаря. Соответственно, размерность вектора определяется количеством стем из словаря. Разобьем третье предложение на токены, т.е. на уникальные слова, и выделим стемы. Если стем из словаря присутствует в предложении, то элемент вектора этого стема будет равен 1 , в противном случае - 0 .

Далее этот вектор подается на вход нейронной сети и на выходе получаем два нейрона: первый отвечает за долю позитивного текста, второй - за долю негативного.

Развитие нейронных сетей повлекло за собой появление в 2015 г. крупных модулей с нейросетями - TensorFlow и Keras [13].

\section{2. Анализ тональности текста с использованием модуля TensorFlow}

Проанализируем эмоциональную окраску текста с помощью модуля TensorFlow. Для обучения нейронной сети понадобятся базы данных с позитивными и негативными текстами. В качестве таких текстов воспользуемся русскоязычными постами микроблогинговой площадки Twitter [14], которые были собраны в 2014 г.

В качестве входных данных будем использовать 7 предложений:

1. Давай дружить.

2. Я люблю тебя.

3. Ты очень красивый.

4. У меня все прекрасно.

5. На улице играют дети.

6. В школе ребенок подрался с одноклассником.

7. Какая у тебя ужасная внешность!

Последние два предложения явно негативные, а остальные - нейтральные или положительные. В дальнейшем определим уровень негативности этих предложений [15].

Порядок анализа текста, с помощью этого модуля, выполняется по следующему алгоритму:

1. Импортирование необходимых модулей.

2. Загрузка данных из баз с негативными и позитивными твитами.

3. Создание стеммера (функции, определяющей стем слова), где regex отсеивает смайлы и символы в твитах (рис. 1). 


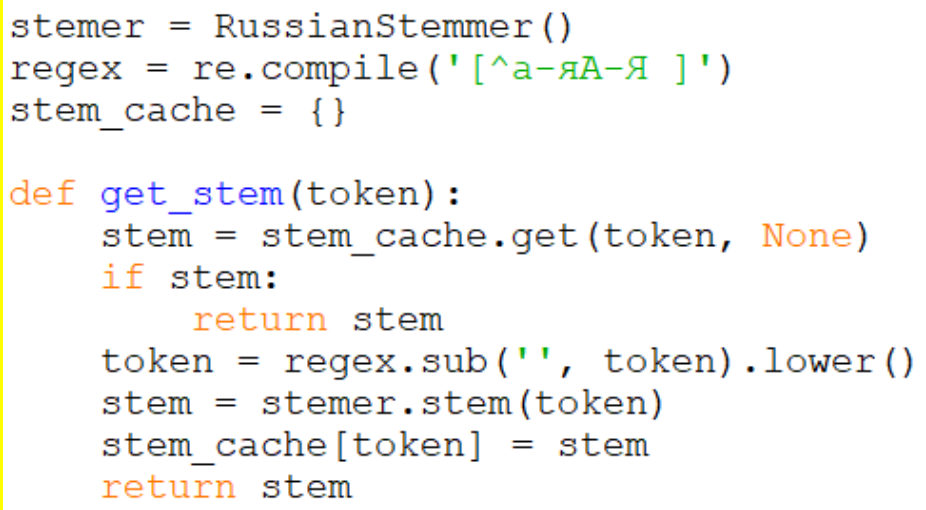

Рис. 1. Программный код создания стеммера

Fig. 1. The program code for creating a stemmer твитов.

4. Формирование словарей с уникальными словами из позитивных и негативных

5. Формирование словаря, который используется для преобразования токена в число.

6. Определение функции преобразования твита в вектор, который подается в нейронную сеть (рис. 2).

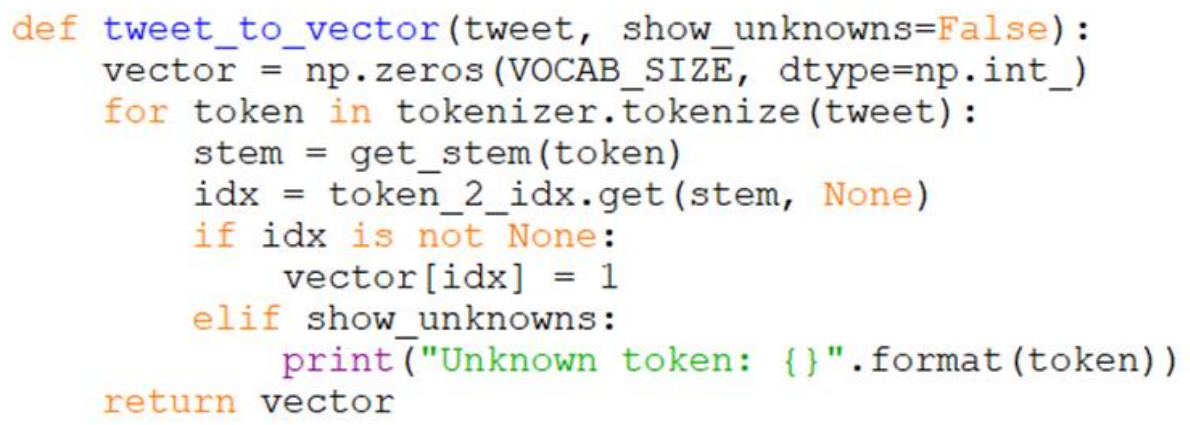

Рис. 2. Программный код определения функиии преобразования твита в вектор

Fig. 2. The program code for defining a function for converting a tweet into a vector

7. Формирование матрицы из преобразованных твитов в векторы.

8. Формирование выходного вектора (рис. 3).

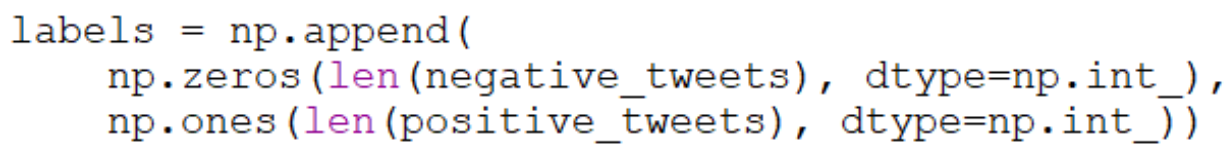

Рис. 3. Программный код формирования выходного вектора

Fig. 3. The program code for formation of the output vector

9. Формирование данных для обучения.

10. Построение нейронной сети (рис. 4). 


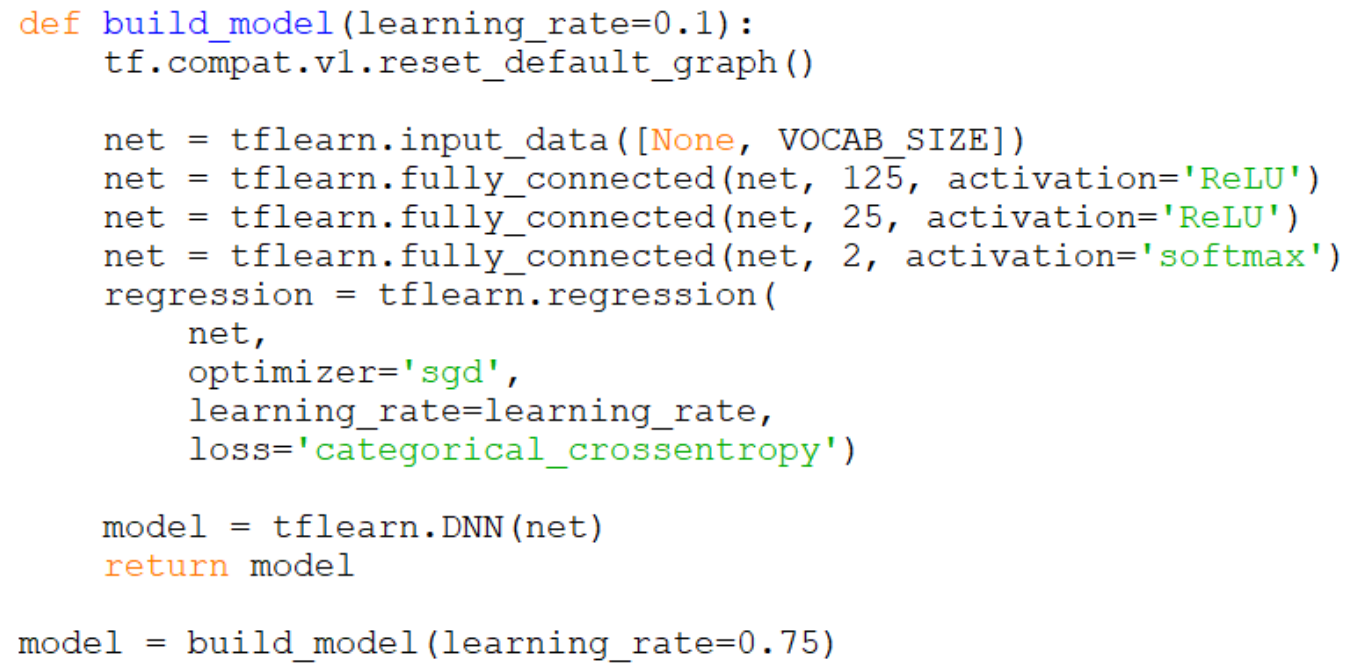

Рис. 4. Программный код построения нейронной сети

Fig. 4. The program code for building a neural network

11. Обучение и тестирование нейронной сети (рис. 5).

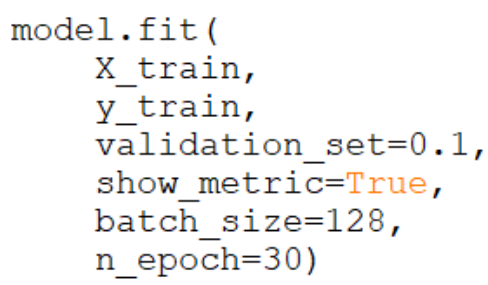

Рис. 5. Программный код обучения и тестирования нейронной сети

Fig. 5. The program code for training and testing a neural network

12. Анализ тональности входящих высказываний и вывод уровня негативности у каждого высказывания (рис. 6).

Результаты сентимент-анализа:

1. Давай дружить - низкий уровень негативности текста.

2. Я люблю тебя - средний уровень негативности текста.

3. Ты очень красивый - низкий уровень негативности текста.

4. У меня все прекрасно - низкий уровень негативности текста.

5. На улице играют дети - высокий уровень негативности текста.

6. В школе ребенок подрался с одноклассником - критический уровень негативности текста.

7. Какая же у тебя ужасная внешность! - критический уровень негативности текста. 


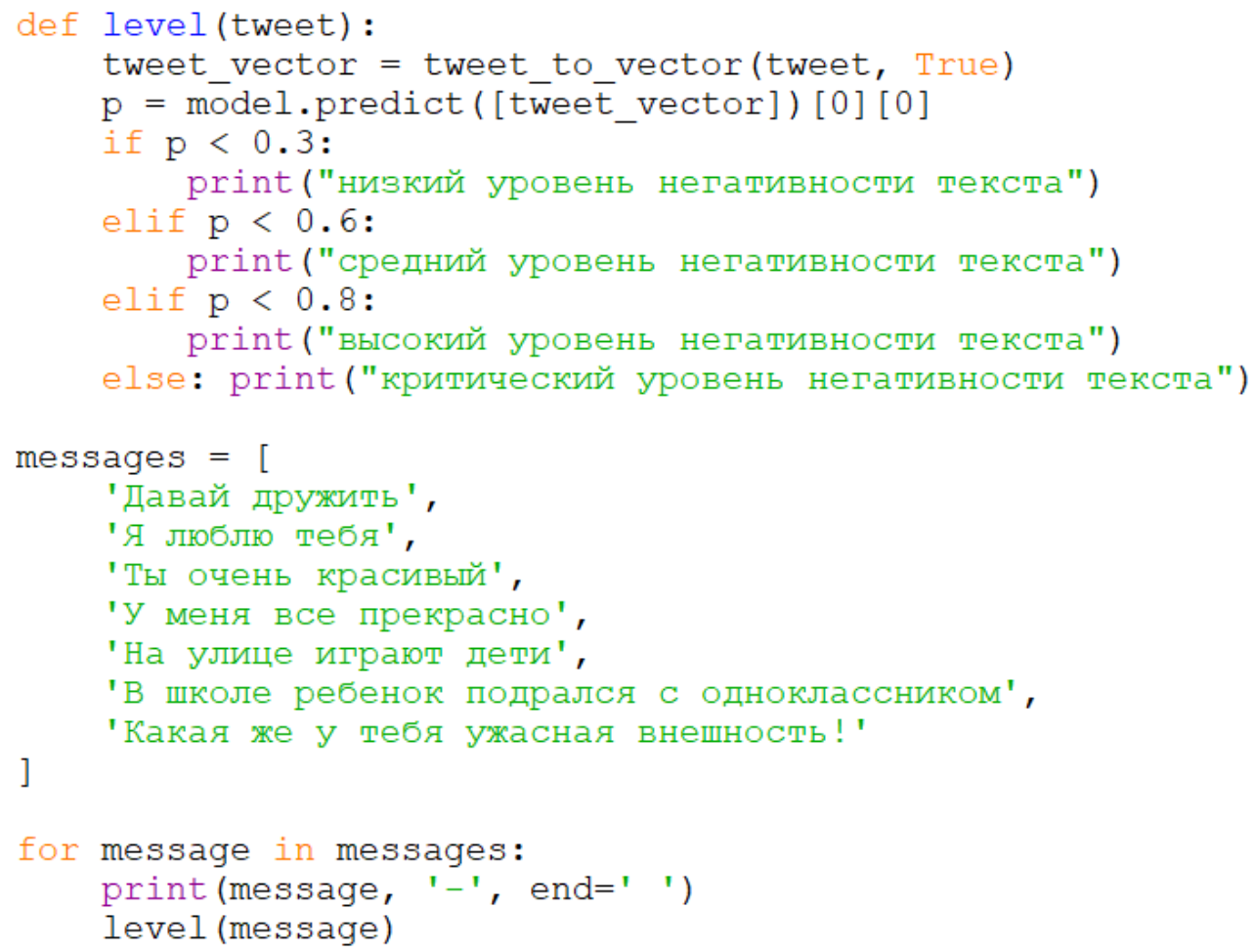

Рис. 6. Программный код анализа тональности входящих высказываний (теssages) и вывод результатов анализа

Fig. 6. The program code for sentiment analysis of incoming messages and output of analysis results

Анализ данных результатов показал - у пяти из семи предложений программный код верно определил оценку тональности. Следовательно, вероятность получения правильного уровня негативности текста при использовании программного кода сентимент-анализа с помощью модуля TensorFlow равна $\mathrm{P}_{1}=5 / 7 \approx 0,71$.

\section{3. Анализ тональности текста с использованием модуля Dostoevsky}

Проанализируем эмоциональную окраску текста с помощью модуля Dostoevsky Данный модуль использует уже обученную нейронную сеть и датасет, содержащий посты из социальной сети ВКонтакте за 2018 г. При этом посты из данной базы категорированы на пять типов:

- negative (негативное настроение);

- positive (позитивное настроение);

- neutral (нейтральное поведение);

- speech (разговорный формат, в который входят поздравления, благодарственные посты, приветствия);

- skip (непонятные случаи, когда у текста нельзя определить настроение).

На вход программного кода, основанного на данном модуле, подаются предложения, использованные в предыдущем программном коде [11] (рис. 7).

После выполнения программного кода получаем следующие уровни негативности предложений:

1. Давай дружить - низкий уровень негативности текста.

2. Я люблю тебя - низкий уровень негативности текста.

3. Ты очень красивый - низкий уровень негативности текста. 
4. У меня все прекрасно - низкий уровень негативности текста.

5. На улице играют дети - низкий уровень негативности текста.

6. В школе ребенок подрался с одноклассником - низкий уровень негативности текста.

7. Какая же у тебя ужасная внешность! - критический уровень негативности текста.

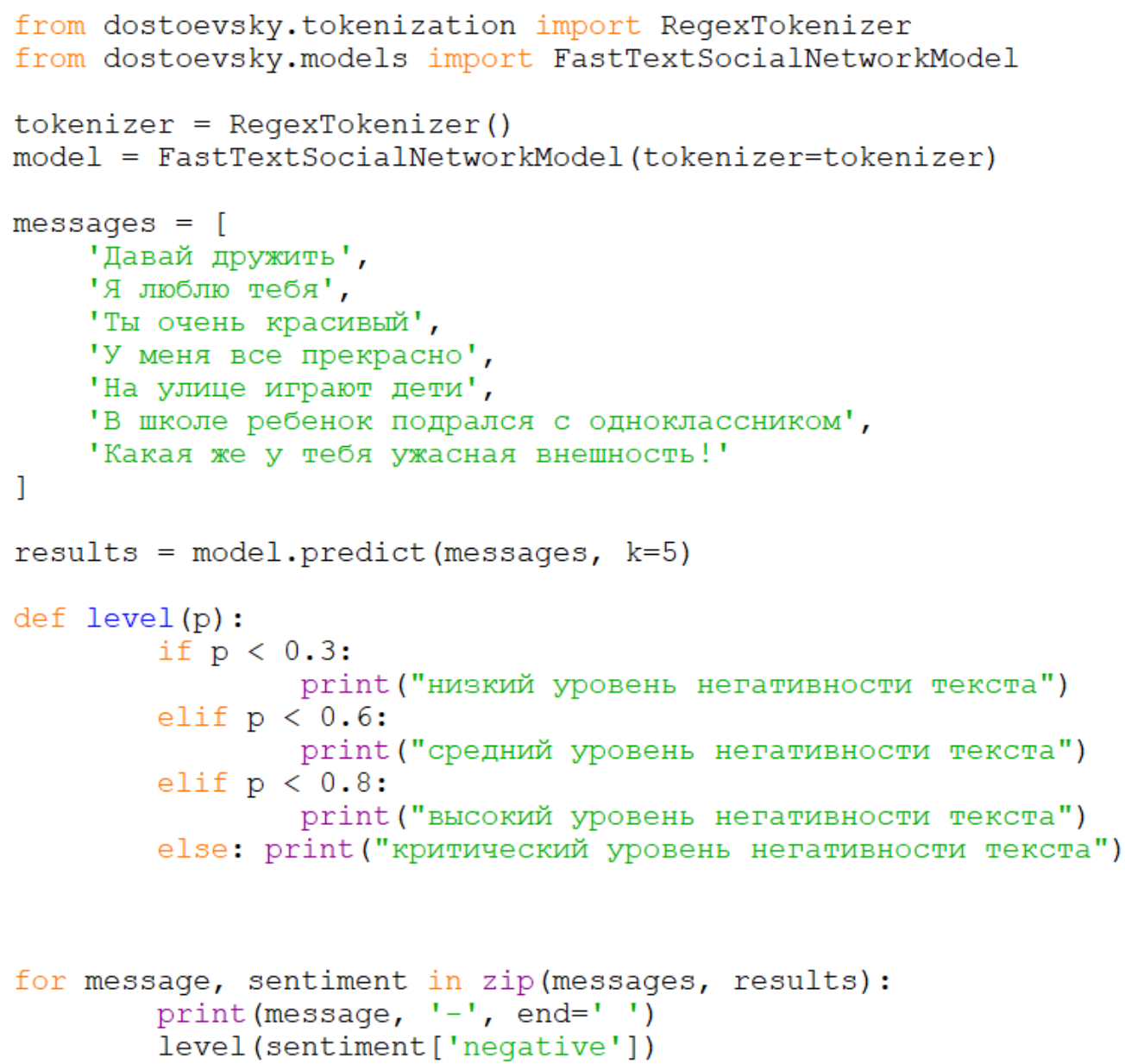

Pис. 7. Программный код анализа тональности текста с помощью модуля Dostoevsky Fig. 7. Program code for text sentiment analysis using the Dostoevsky module

Данный программный код анализа тональности текста с помощью модуля Dostoevsky достаточно правильно оценил предложения на позитивность и негативность верно определил оценку тональности у шести из семи предложений. Ошибка с определением негативности только у шестого предложения. Анализ показывает, что вероятность получения правильного уровня негативности текста равна $\mathrm{P}_{2}=6 / 7 \approx 0,86$.

\section{4. Сравнение результатов работы программных кодов}

Результаты выполнения двух программных кодов анализа текста (в первом коде использовался модуль TensorFlow, в которой нейронная сеть обучается на базах данных 2014 г., содержащие позитивные и негативные тексты, во втором коде - модуль Dostoevsky, в котором нейронная сеть была обучена на базе данных 2018 г., классифицирующихся на 5 категорий) приведены в табл. 1. 
Владимир Л. Евсеев, Руфия Ш. Садекова

ПРОТИВОДЕЙСТВИЕ КИБЕРБУЛЛИНГУ В СОЦИАЛЬНЫХ СЕТЯХ

Таблица 1. Результаты выполнения программных кодов анализа текста

\begin{tabular}{|c|c|c|}
\hline Предложение & $\begin{array}{c}\text { Уровень негативности } \\
\text { текста, определенный } \\
\text { с помощью первого } \\
\text { программного кода } \\
\left(\mathrm{P}_{1} \approx 0,71\right)\end{array}$ & $\begin{array}{c}\text { Уровень негативности } \\
\text { текста, определенный } \\
\text { с помощью второго } \\
\text { программного кода } \\
\left(\mathrm{P}_{2} \approx 0,86\right)\end{array}$ \\
\hline Давай дружить & Низкий & Низкий \\
\hline Я люблю тебя & Средний & Низкий \\
\hline Ты очень красивый & Низкий & Низкий \\
\hline У меня все прекрасно & Низкий & Низкий \\
\hline На улице играют дети & Высокий & Низкий \\
\hline $\begin{array}{c}\text { В школе ребенок подрался } \\
\text { с одноклассником }\end{array}$ & Критический & Критический \\
\hline $\begin{array}{c}\text { Какая же у тебя ужасная } \\
\text { внешность }\end{array}$ & Критический & \\
\hline
\end{tabular}

По результатам анализа табл. 2 можно сделать вывод: наиболее достоверным является программный код с модулем Dostoevsky, так как вероятность получения верного уровня негативности у предложений среди других полученных вероятностей является максимальной $\left(\mathrm{P}_{2} \approx 0,86\right)$. Это связано с тем, что данный модуль использует более актуальную базу данных, содержащую наиболее современные слова в текстах, и обученную ею нейронную сеть. Также в результате выполнения программного кода с использованием модуля Dostoevsky можно получить оценку тональности предложений по 5 категориям, что позволяет точнее определить эмоциональную окраску входящего текста.

\section{Заключение}

В работе показано, что для защиты от кибербуллинга, пользователям сети Интернет целесообразно, в дополнение к организационным и правовым мерам защиты, использовать технические меры защиты - блокировщики сайтов. В основе их работы лежит программа блокирования URL-адресов сайтов с текстом негативного характера. Алгоритм разработанной программы основан на анализе тональности текста (сентиментанализ), позволяющий определять показатели и уровни негативности. Важнейшей частью разработанной программы является модуль анализа тональности текста, определяющий уровень негативности, который определяет эффективность работы блокировщика сайтов. Проведено сравнение результатов выполнения двух программных кодов сентиментанализа предложений (первый программный код основан на модуле TensorFlow, второй на модуле Dostoevsky), сделан вывод о целесообразности применения модуля Dostoevsky, использующего обученную нейронную сеть и усовершенственную базу данных, содержащую пять типов категорий.

В дальнейшем целесообразно использовать для обучения нейронных сетей датасеты, собранные государственными структурами, которые должны периодически обновляться новыми текстами, содержащими актуальную лексику. Это связано с увеличением количества неологизмов русского языка, чему способствует повышение значимости зарубежных социальных сетей.

\section{СПИСОК ЛИТЕРАТУРЫ:}

1. Volkova O.V., Shesternya P.A. Parallel pandemic: psychological facilitation in medical assistance // Сибирский психологический журнал. $2020 . \quad$ № $\quad 78 . \quad$ С. $156-167$. URL: https://cyberleninka.ru/article/n/parallel-pandemic-psychological-facilitation-in-medical-assistance (дата обращения: 02.03.2021). DOI: http://dx.doi.org/10.17223/17267080/78/10. 
2. Мацкевич И.М., Бочкарева Е.В. Пандемия и криминализация общества: как помешать появлению порочного круга. Часть 1 // Мониторинг правоприменения. 2020. URL: https://cyberleninka.ru/article/n/pandemiya-i-kriminalizatsiya-obschestva-kak-pomeshat-poyavleniyuporochnogo-kruga-chast-1 (дата обращения: 02.03.2021). DOI: http://dx.doi.org/10.21681/2226-0692-20203-81-87.

3. Sobkin V.S., Fedotova A.V. Teenagers in social networks: patterns of usage and aggressiveness // Journal of Siberian Federal University. Humanities \& Social Sciences. 2019. № 9. P. 1733-1752. URL: https://cyberleninka.ru/article/n/teenagers-in-social-networks-patterns-of-usage-and-aggressiveness (дата обращения: 02.03.2021). DOI: http://dx.doi.org/10.17516/1997-1370-0480.

4. Зинцова А.С. Социальная профилактика кибербуллинга. Вестник Нижегородского университета им. Н.И. Лобачевского. 2014.2 № $3 . \quad$ С. URL: https://cyberleninka.ru/article/n/sotsialnaya-profilaktika-kiberbullinga (дата обращения: 02.03.2021).

5. Волкова Е.Н., Цветкова Л.А., Волкова И.В. Методологические основания для разработки программ профилактики подросткового буллинга // Сибирский психологический журнал. 2019. № 74. С. 88-100. URL: https://cyberleninka.ru/article/n/metodologicheskie-osnovaniya-dlya-razrabotki-programm-profilaktikipodrostkovogo-bullinga (дата обращения: 07.03.2021). DOI: http://dx.doi.org/10.17223/17267080/74/5.

6. Барей Н.С., Мальцева В.А. Информационная безопасность детей в виртуальном пространстве. Кибербуллинг как стигматизационный фактор // Теория и практика общественного развития. 2020. №1 (143). C. 102-106. URL: https://cyberleninka.ru/article/n/informatsionnaya-bezopasnost-detey-vvirtualnom-prostranstve-kiberbulling-kak-stigmatizatsionnyy-faktor-1 (дата обращения: 02.03.2021).

7. Пахонина Е.В. К проблеме дистанционной коммуникации. Международный научно-исследовательский журнал. 2020. № 9-2 (99). URL: https://cyberleninka.ru/article/n/k-probleme-distantsionnoy-kommunikatsii (дата обращения: 04.03.2021). DOI: http://dx.doi.org/10.23670/IRJ.2020.99.9.048.

8. McMahon R., Bennett I. U.S. Internet Providers and the "Great Firewall of China» // Council on Foreign Relations. 23.02.2011. URL: https://www.cfr.org/backgrounder/us-internet-providers-and-great-firewall-china (дата обращения: 10.03.2021).

9. Shah A. Sentiment analysis of product reviews using supervised learning // RT\&A. 2021. №SI 1 (60). P. 243-253. URL: https://cyberleninka.ru/article/n/sentiment-analysis-of-product-reviews-using-supervisedlearning (дата обращения: 07.03.2021).

10. Sak H., Senior A., Beaufays F. Long short-term memory recurrent neural network architectures for large-scale acoustic modeling // Proceedings of the Annual Conference of the International Speech Communication Association. $2014 . \quad$ P. $\quad 338-342 . \quad$ URL: speech.org/archive/archive_papers/interspeech_2014/i14_0338.pdf (дата обращения: 07.03.2021).

11. Силаева А.Э., Габриелян Г.А., Исаева И.А., Никульчев Е.В. Интеллектуальный анализ текстовых ответов в массовых опросах // Cloud of Science. 2019. Т. 6. № 4. C. 779-788. URL: $\quad$ https://cyberleninka.ru/article/n/intellektualnyy-analiz-tekstovyh-otvetov-v-massovyh-oprosah (дата обращения: 07.03.2021).

12. Rogers A., Romanov A., Rumshisky A., Volkova S., Gronas M., Gribov A. RuSentiment: An Enriched Sentiment Analysis Dataset for Social Media in Russian // Proceedings of the 27th International Conference on Computational Linguistics. 2018. P. 755-763. URL: https://www.aclweb.org/anthology/C18-1064.pdf (дата обращения: 07.03.2021).

13. Druki A.A., Spitsyn V.G., Arkalykov E.U. Semantic segmentation algorithms of the earth's surface pictures based on neural network methods // Вестник Томского государственного университета. Управление, вычислительная техника и информатика. 2020. № 51. URL: https://cyberleninka.ru/article/n/algoritmysemanticheskoy-segmentatsii-snimkov-zemnoy-poverhnosti-na-osnove-neyronnyh-setey (дата обращения: 07.03.2021). DOI: http://dx.doi.org/10.17223/19988605/51/8.

14. Рубцова Ю.В. Построение корпуса текстов для настройки тонового классификатора // Программные $\begin{array}{llllllll}\text { продукты и } & \text { системы. } 2015 . & \text { № } & 1 & \text { (109). } & \text { С. 72-78. }\end{array}$ URL: http://www.swsys.ru/index.php?page=article\&id=3962\&lang $=\quad$ (дата обращения: 05.03.2021). DOI: http://dx.doi.org/10.15827/0236-235X.109.072-078.

15. Karuchit W. Negative effects of digital media on Thai youngsters: Case studies from Thailand and abroad // International Journal of Media and Information Literacy. 2016. № 2. URL: https://cyberleninka.ru/article/n/negative-effects-of-digital-media-on-thai-youngsters-case-studies-fromthailand-and-abroad (дата обращения: 05.03.2021). DOI: http://dx.doi.org/10.13187/ijmil.2016.2.122.

\section{REFERENCES:}

[1] Volkova O.V., Shesternya P.A. Parallel pandemic: psychological facilitation in medical assistance. Siberian Psychological Journal. 2020. No. 78. P. 156-167. URL: https://cyberleninka.ru/article/n/parallel-pandemic- 
psychological-facilitation-in-medical-assistance

(accessed:

02.03.2021).

DOI: http://dx.doi.org/10.17223/17267080/78/10

[2] Matskevich I.M., Bochkareva E.V. Pandemic and criminalization of society: how to prevent the emergence of a vicious circle. Part 1. Monitoring enforcement. 2020. URL: https://cyberleninka.ru/article/n/pandemiya-ikriminalizatsiya-obschestva-kak-pomeshat-poyavleniyu-porochnogo-kruga-chast-1 (accessed: 02.03.2021). DOI: http://dx.doi.org/10.21681/2226-0692-2020-3-81-87 (in Russian).

[3] Sobkin V.S., Fedotova A.V. Teenagers in social networks: patterns of usage and aggressiveness. Journal of Siberian Federal University. Humanities \& Social Sciences. 2019. No. 9. P. 1733-1752. URL: $\quad$ https://cyberleninka.ru/article/n/teenagers-in-social-networks-patterns-of-usage-and-aggressiveness (accessed: 02.03.2021). DOI: http://dx.doi.org/10.17516/1997-1370-0480.

[4] Zintsova A.S. Social prevention of cyberbullying. Vestnik Nizhegorodskogo universiteta im. N.I. Lobachevsky. 2014. No. 3. P. 122-128. URL: https://cyberleninka.ru/article/n/sotsialnaya-profilaktikakiberbullinga (accessed: 02.03.2021) (in Russian).

[5] Volkova E.N., Tsvetkova L.A., Volkova I.V. Methodological grounds for the development of programs for the prevention of teenage bullying. Siberian psychological journal. 2019. No. 74. P. 88-100. URL: https://cyberleninka.ru/article/n/metodologicheskie-osnovaniya-dlya-razrabotki-programm-profilaktikipodrostkovogo-bullinga (accessed: 07.03.2021). DOI: http://dx.doi.org/10.17223/17267080/74/5 (in Russian).

[6] Barei N.S., Maltseva V.A. Information security of children in the virtual space. Cyberbullying as a stigmatization factor. Theory and practice of social development. 2020. No. 1 (143). P. $102-106$. URL: $\quad$ https://cyberleninka.ru/article/n/informatsionnaya-bezopasnost-detey-v-virtualnom-prostranstvekiberbulling-kak-stigmatizatsionnyy-faktor-1 (accessed: 02.03.2021) (in Russian).

[7] Pakhonina E.V. On the problem of remote communication. International Scientific Research Journal. 2020. No. 9-2 (99). URL: https://cyberleninka.ru/article/n/k-probleme-distantsionnoy-kommunikatsii (accessed: 04.03.2021). DOI: https://doi.org/10.23670/IRJ.2020.99.9.048 (in Russian).

[8] McMahon R., Bennett I. U.S. Internet Providers and the "Great Firewall of China». Council on Foreign Relations. 23.02.2011. URL: https://www.cfr.org/backgrounder/us-internet-providers-and-great-firewall-china (accessed: 10.03.2021).

[9] Shah A. Sentiment analysis of product reviews using supervised learning. RT\&A. 2021. No. SI 1 (60). P. 243-253. URL: https://cyberleninka.ru/article/n/sentiment-analysis-of-product-reviews-using-supervisedlearning (дата обращения: 07.03.2021).

[10] Sak H., Senior A., Beaufays F. Long short-term memory recurrent neural network architectures for large-scale acoustic modeling. Proceedings of the Annual Conference of the International Speech Communication Association. $2014 . \quad$ P. 338-342. URL: speech.org/archive/archive_papers/interspeech_2014/i14_0338.pdf (accessed: 07.03.2021).

[11] Silaeva A.E., Gabrielyan G.A., Isaeva I.A., Nikulchev E.V. Intelligent analysis of text responses in mass polls. Cloud of Science. 2019. Vol. 6 No. 4. P. 779-788. URL: https://cyberleninka.ru/article/n/intellektualnyyanaliz-tekstovyh-otvetov-v-massovyh-oprosah (accessed: 07.03.2021) (in Russian).

[12] Rogers A., Romanov A., Rumshisky A., Volkova S., Gronas M., Gribov A. RuSentiment: An Enriched Sentiment Analysis Dataset for Social Media in Russian. Proceedings of the 27th International Conference on Computational Linguistics. 2018. P. 755-763. URL: https://www.aclweb.org/anthology/C18-1064.pdf (accessed: 07.03.2021).

[13] Druki A.A., Spitsyn V.G., Arkalykov E.U. Semantic segmentation algorithms of the earth's surface pictures based on neural network methods // Bulletin of Tomsk State University. Management, Computer Engineering and Informatics. 2020. No. 51. URL: https://cyberleninka.ru/article/n/algoritmy-semanticheskoy-segmentatsiisnimkov-zemnoy-poverhnosti-na-osnove-neyronnyh-setey (accessed:

07.03.2021) DOI: http://dx.doi.org/10.17223/19988605/51/8.

[14] Rubtsova Yu. Building a text corpus for tuning a tone classifier // Software products and systems. 2015. No. 1 (109). P. 72-78. URL: http://www.swsys.ru/index.php?page=article\&id=3962\&lang= (accessed: 05.03.2021). DOI: http://dx.doi.org/10.15827/0236-235X.109.072-078 (in Russian).

[15] Karuchit W. Negative impact of digital media on Thai youth: Case studies from Thailand and abroad. International Journal of Media and Information Literacy. 2016. No. 2. URL: https://cyberleninka.ru/article/n/negative-effects-of-digital-media-on-thai-youngsters-case-studies-fromthailand-and-abroad (accessed: 05.03.2021). DOI: http://dx.doi.org/10.13187/ijmil.2016.2.122.

Поступила в редакичю - 29 марта 2021 г. Окончательный вариант - 25 Августа 2021 . Received-March 29, 2021. The final version-August 20, 2021. 